\title{
Revisión
}

\section{Cáncer de riñón hereditario}

\author{
Julián Sanz-Ortega, Carlos Olivier*, Pedro Pérez Segura**, Isabel Galante Romo*, \\ Luis San José Mansó*, Mamen Saez
}

\author{
Servicio de Anatomía Patológica, *Urología y **Oncología. Hospital Clínico San Carlos. Madrid, España \\ Unidad Clínica del Cáncer Renal Hereditario (UCCRH) (Integrado en la Comisión multidisciplinar de Consejo \\ Genético Hospital Clínico San Carlos)
}

\section{Resumen}

El cáncer de riñón es la décima causa más común de muerte por cáncer. Esta revisión analiza las características de los síndromes hereditarios más frecuentes asociados a un subtipo histológico de tumor renal específico, su prevalencia y penetrancia, test genéticos disponibles y programas de cribado/detección precoz y tratamiento recomendados.

En el síndrome de Von Hippel-Lindau un $40 \%$ de los pacientes desarrollan carcinoma renal de células claras bilateral y multifocal. También son frecuentes el hemangioblastoma del SNC o retina, feocromocitomas y tumores del saco endolinfático. Se han descrito cuatro fenotipos clásicos de VHL en función de la mutación y un diferente riesgo de feocromocitoma o carcinoma de células renales. Se puede realizar test genético de diagnóstico de confirmación, diagnóstico prenatal o preimplantación.

El cáncer papilar renal hereditario tiene múltiples carcinomas papilares bilaterales de subtipo histológico 1. El gen asociado es el proto-oncogen c-met.

El síndrome de Birt-Hogg-Dubé por mutaciones en el gen FLCN combina múltiples tumores renales bilaterales de tipo oncocitoma, carcinoma cromófobo, tumor híbrido oncocítico y una minoría carcinoma de células claras renales. Se asocia a fibrofoliculomas cutáneos, quistes de pulmón y neumotórax espontáneo. Histológicamente, hay lesiones iniciales de oncocitosis o híbridos oncocíticos excepcionales fuera del síndrome hereditario.

La leiomiomatosis hereditaria y cáncer de células renales por mutaciones del gen fumarato hidratasa tiene en un 15\% de los pacientes un agresivo carcinoma papilar tipo 2, en un $75 \%$ leiomiomas cutáneos múltiples y en 100\% leiomiomas uterinos. En el estudio histopatológico se observan unos macronúcleolos eosinófilos característicos.

La Esclerosis tuberosa es uno de los síndromes hereditarios más frecuentes asociado a angiomiolipoma $(70 \%$ de afectados), quistes renales, oncocitoma o carcinoma renal de células claras.

Palabras clave: Cáncer hereditario. Riñón. Consejo genético.

\section{Hereditary renal cancer}

\section{Abstract}

Kidney cancer is the tenth most common cause of cancer death. There are a growing number of genes known to be associated with an increased risk of specific types of kidney cancer.

People with Von Hippel-Lindau syndrome have about a 40\% risk of developing multiple bilateral clear cell kidney cancers. They can also develop retinal and brain hemangioblastoma, kidneys or pancreas cysts, pheochromocytoma and endolymphatic sac tumor. Four phenotypes with different renal cancer and pheocromocitoma risk have been described depending on the germline mutation.

Hereditary papillary renal cell carcinoma syndrome has type 1 papillary renal cell carcinomas associated with protooncogene c-MET germline mutations.

Birt-Hogg-Dubé syndrome has FLCN gene mutations associated with fibrofolliculomas, lung cysts with a high risk for spontaneous pneumothorax, and a 15\% to 30\% risk of kidney cancer (most classified as chromophobe carcinoma, oncocytoma or oncocytic hybrid, but clear cell and papillary kidney cancers have also been reported). Histopathological findings such as oncocytosis and oncocytic hybrids are very unusual outside the syndrome.

Hereditary leiomyomatosis and renal cell cancer syndrome shows mutations of Fumarate hydratase gene and cutaneous leiomyomata in $76 \%$ of affected individuals, uterine leiomyomata in $100 \%$ of females, and unilateral, solitary, and aggressive papillary renal cancer in 10 to $16 \%$ of patients. A specific histopathological change is eosinophilic prominent nucleoli with a perinucleolar halo.

Tuberous sclerosis complex is one of the most prevalent $(1 / 5.800)$ hereditary syndromes where renal disease is the second leading cause of death, associated with angiomyolipomas (70\%), renal cysts, oncocytomas or clear cell cancer. 
$\mathrm{E}^{1}$ cáncer de riñón es el séptimo cáncer por frecuencia en los hombres, noveno en las mujeres, y en conjunto la décima causa más común de muerte por cáncer. En 2008, se estima que se diagnosticarán 54390 nuevos casos de cáncer de riñón (33.130 hombres y 21.260 mujeres) en los Estados Unidos, con una estimación de 13.010 muertes (8.100 hombres y 4.910 mujeres) $^{1}$. En España según el INE la estimación es de 2.000 muertes/año. Algunas de estas muertes podrían evitarse con un mejor manejo de las personas con riesgo aumentado de cáncer renal por predisposición genética.

El cáncer de riñón no es una sola enfermedad, lo componen distintas entidades con comportamiento biológico, morfología y base molecular diferentes. Hay un número creciente de genes que están asociados con un mayor riesgo de cáncer de riñón. Algunos de estos síndromes hereditarios se han descrito muy recientemente y la prevalencia real no se sabe con certeza, se cree que al menos el 5\% de los carcinomas renales tiene base hereditaria. La identificación de un síndrome genético en una familia puede ayudar a desarrollar un plan de cribado del cáncer y, en algunos casos, a determinar el mejor tratamiento.

Esta revisión analiza las características de los síndromes hereditarios más frecuentes, generalmente asociados a un subtipo histológico específico, la frecuencia de tumores, test genéticos disponibles y programas de cribado/detección precoz y tratamiento recomendados.

Los síndromes familiares asociados con distintos tipos de cáncer renal incluyen los siguientes:

- Von Hippel-Lindau (VHL): carcinoma renal de células claras bilateral y multifocal. Las personas con sindrome de VHL están también en situación de riesgo para hemangioblastoma del SNC, angioma de retina, feocromocitoma, y tumores del saco endolinfático.

- Cáncer papilar renal hereditario (HPRC): carcinoma papilar bilateral y multifocal tipo 1 .

Leiomiomatosis hereditaria y cáncer de células renales (HLRCC): tumores renales solitarios tipo carcinoma papilar tipo 2. Los individuos con HLRCC pueden presentar leiomiomas cutáneos múltiples y/o uterinos con inicio temprano y agresivo.

- Birt-Hogg-Dubé: Los tumores renales suelen ser multifocales y bilaterales. Incluyen: Oncocitoma, carcinoma cromófobo, tumor híbrido oncocítico, y una minoría carcinoma de células claras renales. Se asocia a fibrofoliculomas cutáneos.
- Esclerosis tuberosa: asociada a angiomiolipoma.

La mutación heredada en estos síndromes debe estar presente en la línea germinal y por tanto en todas las células. Se recomienda el estudio de sangre periférica por la mayor especificidad, sencillez y rapidez del estudio. También se puede estudiar material de archivo incluido en parafina o congelado de los tumores y tejido normal.

En esta revisión se especifican los criterios clínicos y/o histopatológicos suficientes para en cada síndrome considerar al paciente susceptible de realizar el test genético. Por tanto, la sospecha de sindrome hereditario puede partir de patólogos, urólogos, dermatólogos u otros especialistas, en ocasiones de manera coordinada. El paciente será examinado e informado de las consecuencias y alcance del estudio por el equipo de consejo genético. En caso de no poder desplazarse, si se desea enviar muestra para estudio genético, el primer requisito "imprescindible" es obtener consentimiento informado del paciente (ley de investigación biomédica 14/2007, 3 Julio 2007). El siguiente paso es la extracción de $\mathrm{ADN}$ de la sangre (o en su caso de tejido congelado o incluido en parafina) y análisis molecular, tal y como se describe con posterioridad.

\section{SÍNDROMES HEREDITARIOS \\ Síndrome de Von Hippel-Lindau (VHL)}

Características: Las personas con VHL tienen un mayor riesgo de varios tipos de tumores ${ }^{2-10}$. La mayoría de estos tumores son benignos. Sin embargo, las personas con VHL tienen un $40 \%$ de riesgo de desarrollar cáncer de riñón y ésta es la principal causa de mortalidad. El tipo específico de cáncer de riñón relacionado con VHL es el carcinoma de células claras de riñón. Otras partes del cuerpo donde pueden desarrollar tumores incluyen el ojo (angio$\mathrm{ma}^{5,6}$, el cerebro ${ }^{7}$ y la médula espinal (hemangioblastoma), las glándulas suprarrenales (feocromocitoma), y los oídos (tumor del saco endolinfático ${ }^{8}$. Las personas con VHL pueden también desarrollar quistes en sus riñones o páncreas. Los síntomas de VHL usualmente se desarrollan a los 20 y 30 años, pero pueden aparecer en la infancia. Aproximadamente el 20\% de personas con VHL no tienen historia familiar de la enfermedad.

Diagnóstico: El diagnóstico clínico de síndrome von Hippel-Lindau se establece en ${ }^{2}$ :

Una persona que no refiere o conoce historia familiar requiere la presentación de dos o más lesio- 
nes características: por ejemplo, dos o más hemangioblastomas de la retina o el cerebelo o un solo hemangioblastoma en asociación con una manifestación visceral como quistes de riñón o páncreas, carcinoma de células renales antes de la edad de 60 años, feocromocitomas, y, menos comúnmente, los tumores del saco endolinfático, cistadenoma papilar del epidídimo o de ligamentos anchos, o tumores neuroendocrinos del páncreas ${ }^{9}$.

Una persona con historia familiar de síndrome de VHL requiere sólo una de las manifestaciones anteriores.

Sospecha anatomopatológica: Además de las características anteriores el patólogo puede sospechar el sindrome cuando coinciden de manera sincrónica o metacrónica:

- Espectro de lesiones bilaterales: Carcinomas renal de células claras (CRCC) y quistes renales.

- Lesiones quísticas precursoras y formas iniciales de CRCC.

Gen: VHL es el único gen asociado con el síndrome de VHL (cromosoma 3p25-p26). Se hereda en forma autosómica dominante. VHL actúa como gen supresor de tumores y es un componente fundamental de un complejo proteico que se une y degrada la proteína HIF1 (Factor inductor de hipoxia). Cuando se pierde la función de VHL, HIF1 permanece activo de manera anómala actuando como estimulador de rutas moleculares carcinogenéticas que activan la proliferación celular.

Pruebas de genética molecular/testing ${ }^{10-12}$ : El diagnóstico de síndrome de VHL se sospecha en individuos con las lesiones características. Las pruebas de genética molecular del gen VHL detectan mutaciones en casi el 100\% de los individuos afectados.

Utilidad clínica ${ }^{11,12}$ :

- Pruebas de diagnóstico de confirmación

- Pruebas de predicción

- El diagnóstico prenatal

- Diagnóstico genético de preimplantación

Método habitual: Análisis de secuencias de los tres exones para detectar mutaciones puntuales $(\sim 72 \%$ de mutaciones VHL).

- Diversos métodos (por ejemplo, PCR semicuantitativa) pueden ser usados para detectar pérdida parcial o completa de la región cromosómica del gen que representan aproximadamente el $28 \%$ de todas las mutaciones $\mathrm{VHL}^{12}$.

- Para las personas con manifestaciones de VHL que no cumplen estrictamente los criterios diagnósticos y que no tienen una mutación germinal de VHL detectable, deben considerarse mutaciones somáticas de novo ${ }^{13}$. En algunos casos, las pruebas genéticas moleculares de la descendencia de estas personas pone de manifiesto la mutación VHL.

\section{Correlaciones genotipo-fenotipo ${ }^{14-16}$}

Se han descrito cuatro fenotipos clásicos de VHL basado en el riesgo de feocromocitoma o carcinoma de células renales.

VHL tipo 1 se caracteriza por un bajo riesgo de feocromocitoma y mutaciones missense que perturban el plegamiento de la proteína.

VHL tipo 2 se caracteriza por un alto riesgo de feocromocitoma y otras mutaciones missense. VHL tipo 2 se subdividen a su vez:

- Tipo 2A: bajo riesgo de carcinoma de células renales;

- Tipo 2B: alto riesgo de carcinoma renal, y

- Tipo 2C: con un riesgo de feocromocitoma.

Gestión ${ }^{17}$ : Cirugía renal conservadora ante la sucesión de lesiones múltiples (nefrectomía parcial cuando sea posible) de carcinoma de células claras renales; trasplante renal tras nefrectomía bilateral; eliminar feocromocitomas. Prevención de déficits secundarios tales como pérdida de la audición, pérdida de la visión, y síntomas neurológicos. Vigilancia: exploración oftalmológica anual, comenzando antes de la edad de cinco años; control anual de la presión arterial y medición de catecolaminas en orina que comienza en la edad de cinco años en las familias con una alta incidencia de feocromocitoma; ecografía anual abdominal que comienza en la edad de 16 años con la evaluación de lesiones sospechosas con TAC o resonancia magnética.

Pruebas de familiares en situación de riesgo: aclarar el status genético de los miembros de la familia para eliminar la necesidad de gastos costosos de vigilancia en los miembros de la familia que no han heredado la mutación.

Penetrancia: Casi todas las personas que tienen una mutación en el gen VHL van a manifestar síntomas relacionados con la enfermedad antes de los 65 años. 
Prevalencia: La incidencia de VHL es aproximadamente uno de cada 36000 nacimientos ${ }^{18}$.

\section{Carcinoma hereditario papilar de células renales (HPRCC)}

HPRCC se sospecha cuando dos o más parientes cercanos han sido diagnosticados de carcinoma papilar renal tipo 1. El cáncer renal se diagnostica generalmente a partir de los 40 años en estas familias, sobre todo en la sexta década de la vida. Las personas con HPRCC pueden desarrollar múltiples tumores renales en uno o ambos riñones. Las personas en familias en las que se identifica la mutación de HPRCC deben hacer un programa de cribado anual mediante ultrasonido, resonancia magnética y/o tomografía computarizada con comienzo en torno a los 30 años de edad.

El gen asociado con HPRCC se llama c-met (localizado en el cromosoma 7q31), es un proto-oncogen y por tanto produce proteínas que promueven el crecimiento celular normal. El carcinoma papilar tipo 1 se caracteriza además, tanto en formas familiares como esporádicas, por la trisomía en los cromosomas 7,16 , ó 17, y en los hombres, por la pérdida del cromosoma $\mathrm{Y}^{19}$

El patrón de herencia es compatible con transmisión autosómica dominante con penetrancia baja.

\section{Sindrome de Birt-Hogg-Dubé (BHD)}

Resumen: BHD es un raro síndrome descrito en $1997^{20}$ asociado con fibrofoliculomas (tumores benignos de los folículos pilosos que son generalmente de color blanco o color carne), quistes pulmonares, y un mayor riesgo de cáncer de riñón. Las personas con BHD tienen un 15\% y el 30\% de riesgo de desarrollar cáncer de riñón. La mayoría de los cánceres renales asociados con BHD se clasifican como el tipo carcinoma cromófobo y Oncocitoma, aunque también hay de células claras del riñón y cáncer papilar. Debido al mayor riesgo de cáncer de riñón, el cribado anual con ultrasonido, resonancia magnética, tomografía computarizada debe ser considerado a partir de 25 años.

Características Clínicas ${ }^{20-25}$. Se incluyen manifestaciones cutáneas (fibrofoliculomas, tricodiscomas, y acrocordomas), quistes pulmonares e historia de neumotórax y diversos tipos de tumores renales. La gravedad de la enfermedad puede variar considerablemente. Las lesiones cutáneas suelen aparecer durante la tercera o cuarta década de vida y aumentan de tamaño y número con la edad. Los quistes de pulmón son en su mayoría bilaterales y multifocales, asintomáticos, pero tienen un alto riesgo de neumotórax espontáneo. Aproximadamente el 15\% de las personas con sindrome de BHD desarrollan tumores renales, que son de carácter bilateral y multifocal y generalmente de crecimiento lento; la edad media de diagnóstico del tumor es de 48 años. Los más comunes son: Oncocitoma, carcinoma cromófobo o un híbrido de oncocitoma y cromófobo.

\section{Diagnóstico clínico:}

- Manifestaciones cutáneas múltiples, pequeñas, en forma de pápulas distribuidas por cara, cuello y parte superior del tronco. La triada característica es fibrofoliculomas (hamartomas múltiples del folículo piloso), tricodiscomas, y acrocordomas, pero sólo fibrofoliculomas son específicos para el síndrome de BHD. El diagnóstico dermatológico de Birt-HoggDubé se hace en individuos que tienen cinco o más pápulas con al menos un fibrofoliculoma histológicamente confirmado ${ }^{25}$.

Nota: La biopsia por raspado no suele ser suficiente.

- Quistes de pulmón y neumotórax espontáneo. La mayoría (89\%) de las personas con síndrome de BHD tienen múltiples, quistes pulmonares bilaterales, identificados por TAC de tórax. $\mathrm{El}$ número total de quistes por pulmón individual va de 0 a 166 (media 16). 24\% (48/198) de las personas con síndrome de BHD tenía un historial de uno o más neu$\operatorname{motórax}^{25}$.

- Tumores renales. Los tumores renales suelen ser multifocales y bilaterales. Incluyen en combinación: Oncocitoma, carcinoma cromófobo, tumor híbrido oncocítico, y una minoría carcinoma de células claras renales.

* Nota: La descripción original y el diagnóstico de Birt-Hogg-Dubé sindrome se basa en la patología de la piel. Sin embargo, recientes investigaciones han demostrado que algunos individuos con Birt-HoggDubé podrían presentar sindrome pulmonar y / o tumores renales, sin lesiones cutáneas.

- Sospecha anatomopatológica: Cuando existe combinación de carcinomas cromófobos y oncocitomas, especialmente en presencia de lesiones iniciales "Oncocitosis" o híbridos oncocíticos que son poco frecuentes fuera del síndrome hereditario. 
Diagnóstico/testing ${ }^{26-27}$ : FLCN (BHD) es el único gen asociado con Birt-Hogg-Dubé. Se detectan mutaciones en el gen FLCN en el 84\% de los individuos afectados.

Consejo genético: Se hereda en forma autosómica dominante.

\section{Pruebas de genética molecular:}

- Análisis de Secuencias. En un 53\% (27 de 51) de las familias con Birt-Hogg-Dubé se encontró una inserción o deleción en el exón 11 (mutacional hotspot) ${ }^{28}$.

EL análisis de secuenciación de todos los exones (exón 4-14) aumenta la detección de mutaciones en a $84 \%$.

Correlaciones Genotipo-fenotipo: Las personas que tienen una deleción del exón 11 pueden tener un menor riesgo de cáncer renal que las personas con otras mutaciones ${ }^{28}$

Penetrancia: Sobre la base de las tres grandes manifestaciones clínicas, penetrancia de Birt-HoggDubé se considera muy elevada.

Prevalencia: Se han descrito más de 60 familias $\operatorname{afectadas}^{29}$.

\section{Leiomiomatosis herditaria y carcinoma de células renales (HLRCC)}

Resúmen ${ }^{30-35}$ : HLRCC es un síndrome descrito en 2001, cuya incidencia renal se desconoce ${ }^{30}$. Un $76 \%$ de las personas con HLRCC van a desarrollar nódulos cutáneos llamados leiomiomas. Los nódulos se encuentran principalmente en los brazos, piernas, pecho y espalda. Las mujeres con HLRCC desarrollan leiomiomas uterinos (100\%) o, menos comúnmente, leiomiosarcoma. Las personas con HLRCC también tienen un riesgo del 20\% de desarrollo de carcinoma papilar renal tipo 2. Las mujeres en estas familias también deben someterse a las pruebas de estudio ginecológico ya que los leiomiomas uterinos suelen ser sintomáticos y dan lugar a histerectomías precoces (edad media 30 años). El gen asociado con HLRCC se llama FH (fumarato hidratasa).

Diagnóstico clínico ${ }^{35}$ : No hay un consenso sobre los criterios diagnósticos para HLRCC. La sospecha clínica puede provenir entre otros de dermatólogos, urólogos o patólogos en los siguientes supuestos:
- Múltiples leiomiomas cutáneos con al menos un leiomioma confirmado histológicamente

- Un solo leiomioma en presencia de una historia familiar positiva de HLRCC.

- Uno o más carcinoma papilar renal tipo 2 con o sin antecedentes familiares de HLRCC.

- Características histopatológicas típicas en carcinomas papilares renales tipo 2 y/o leiomiomas uterinos: presencia de macronucleolos eosinófilos rodeados de halo claro.

Nota: Debido a que la prevalencia de leiomiomas uterino en la población general es alta, un leiomioma uterino solitario no es suficiente para el diagnóstico de HLRCC.

Estrategia test genético ${ }^{31-32}$ : HLRCC se hereda en forma autosómica dominante. Las pruebas genéticas para detectar generalmente por secuenciación directa de todos los exones una mutación germinal del gen $\mathrm{FH}$.

La medición de la actividad de la enzima fumarato hidratasa puede ser útil en el diagnóstico de HLRCC en los casos con presentación atípica y mutaciones indetectables $\mathrm{FH}$.

Correlaciones Genotipo-fenotipo: No se han descrito correlaciones genotipo-fenotipo. Las mutaciones asociadas con HLRCC se distribuyen a lo largo del gen $\mathrm{FH}$.

Penetrancia: Sobre la base de tres grandes manifestaciones clínicas, de penetrancia HLRCC se considera muy elevada.

Prevalencia: El sindrome se ha descrito recientemente y se desconoce. Hay más de 100 familias con HLRCC descritas.

\section{Esclerosis tuberosa}

Características $^{36,37}$ : La esclerosis tuberosa (ET) implica alteraciones de la piel (maculas hipopigmentadas, angiofibromas faciales, placas fibrosas faciales, fibroma ungueal), cerebro (placas corticales, nódulos subependimarios, convulsiones, retraso mental o retraso en el desarrollo), riñón (angiomiolipomas, quistes), y el corazón (rabdomiomas, arritmias). Los tumores del SNC son la principal causa de morbilidad y mortalidad, mientras que la enfermedad renal es la segunda causa de muerte prematura.

Riñones. La enfermedad renal es la segunda causa de muerte prematura $(27,5 \%)$ en personas con 
esclerosis tuberosa ${ }^{38}$. Se estima que el $80 \%$ de los niños con esclerosis tuberosa tiene una lesión renal identificable de la edad media de 10,5 años. Cinco lesiones renales diferentes se producen en ET: angiomiolipoma benigno (70\% de los individuos afectados), quistes epiteliales (20-30\%), Oncocitoma (hamartoma adenomatoso benigno) $(<1 \%)$, angiomiolipoma malignos $(<1 \%)$ y carcinoma de células renales $(<3 \%)$.

Diagnóstico/testing: El diagnóstico de ET se basa en los hallazgos clínicos. Se han identificado dos genes causales: TSC1 y TSC2 $2^{38-39}$.

Diagnóstico clínico: Los criterios diagnósticos de esclerosis tuberosa (ET) se han revisado ${ }^{40}$.

Consejo genético ${ }^{38-39}$. ET se hereda en forma autosómica dominante. Dos tercios de las personas afectadas tienen ET como el resultado de mutación de novo genética. $27 \%$ de mutaciones en TSC1 y el 73\% con mutaciones en TSC2.

Pruebas de genética molecular:

- Análisis de Secuencias. Las mutaciones de TSC1 son principalmente pequeñas inserciones y deleciones y mutaciones non-sense; por el contrario, las mutaciones de TSC2 también pueden incluir un número significativo de grandes reordenamientos y grandes deleciones que no se pueden detectar por análisis de secuencias.

Las pruebas de genética molecular de TSC1 y TSC2 genes son complicadas por el gran tamaño de los dos genes, el gran número de enfermedades que causan mutaciones, y la elevada tasa de mosaicismo somático $(10-25 \%)^{46}$.

- Genómica-análisis de microarrays/FISH: (SignatureChip ${ }^{\mathrm{TM}}$ ) incluye la evaluación de 125 loci clínicamente relevantes utilizando 589 clones BAC para detectar la presencia o no de la región a prueba de ADN.20-30\% de las personas con esclerosis tuberosa no tienen una mutación identificable $\mathrm{y}$, por tanto, no puede calificarse de subtipo genético.

Correlaciones genotipo-fenotipo: Parece que las mutaciones TSC1 suelen producir un fenotipo menos severo que mutaciones en TSC2 -Al-Saleem et al $(1998)^{37}$ reportaron un mayor riesgo de neoplasia renal en personas con mutaciones en TSC2.

Penetrancia: La penetrancia del TSC parece ser del $100 \%$.

Prevalencia: La incidencia de TSC puede ser tan alta como uno en $5.800^{36}$.

\section{REFERENCIAS}

1. Ries LAG, Melbert D, Krapcho M, Stinchcomb DG, Howlader N, Horner MJ, Mariotto A, Miller BA, Feuer EJ, Altekruse SF, Lewis DR, Clegg L, Eisner MP, Reichman M, Edwards BK (eds). SEER Cancer Statistics Review, 1975-2005, National Cancer Institute. Bethesda, MD, http://seer.cancer.gov/csr/1975_ 2005/, based on November 2007 SEER data submission, posted to the SEER web site, 2008.

2. Linehan WM, Zbar B, Klausner DR. Renal carcinoma. In: Scriver CR, Beaudet AL, Sly WS, Valle D, Vogelstein B (eds) The Metabolic and Molecular Bases of Inherited Disease (OMMBID), McGraw-Hill, New York, Chap 41,2002

3. Molino D, Sepe J, Anastasio P, De Santo NG. The history of von Hippel-Lindau disease. J Nephrol 19 Suppl. 2006; 10: S119-23.

4. Glasker S. Central nervous system manifestations in VHL: genetics, pathology and clinical phenotypic features. Fam Cancer. 2005; 4:37-42.

5. Kreusel KM. Ophthalmological manifestations in VHL and NF 1: pathological and diagnostic implications. Fam Cancer. 2005;4: 43-47.

6. Webster AR, Maher ER, Moore AT. Clinical characteristics of ocular angiomatosis in von Hippel-Lindau disease and correlation with germline mutation. Arch Ophthalmol. 1999;117:371-378.

7. Iliopoulos O. von Hippel-Lindau disease: genetic and clinical observations. Front Horm Res. 2001;28:131-166.

8. Giannini C, Scheithauer BW, Davis DH. Peripheral nerve hemangioblastoma. Mod Pathol. 1998;11:999-1004.

9. Choo D, Shotland L, Mastroianni M, Linehan WM, Oldfield EH. Endolymphatic sac tumors in von Hippel-Lindau disease. J Neurosurg. 2004;100: 480-487.

10. Marcos HB, Libutti SK, Alexander HR, Lubensky IA, Bartlett DL, Walther MM, Linehan WM, Glenn GM, Choyke PL. Neuroendocrine tumors of the pancreas in von HippelLindau disease: spectrum of appearances at CT and MR imaging with histopathologic comparison. Radiology. 2002;225: 751-758.

11. Beroud C, Joly D, Gallou C, Staroz F, Orfanelli MT, Junien C. Software and database for the analysis of mutations in the VHL gene. Nucleic Acids Res. 1998;26:256-258.

12. Rasmussen A, Nava-Salazar S, Yescas P, Alonso E, Revuelta R,Ortiz I,Canizales-Quinteros S,Tusié-Luna MT, López-López M: Von Hippel-Lindau disease germline mutations in Mexican patients with cerebellar hemangioblastoma.J Neurosurg 2006 Mar; 104(3):389-394.

13. American Society of Clinical Oncology. ASCO policy statement update: genetic testing for cancer susceptibility. J Clin Oncol. 2003; 21: 2397-2406.

14. Sgambati MT, Stolle C, Zbar B, Linehan WM, Glenn GM. Mosaicism in von Hippel-Lindau disease: lessons from kindreds with germline mutations identified in offspring with mosaic parents. Am J Hum Genet. 2000;66:84-91.

15. Zbar B, Kishida Tet al. Germline mutations in the (VHL) gene in families from North America, Europe, and Japan. Hum Mutat. 1996;8:348-357.

16. Stebbins CE, Pavletich NP. Structure of the VHL-ElonginCElonginB complex: implications for VHL tumor suppressor function. Science. 1999;284:455-461.

17. Maher ER, Greenstein RM. The von Hippel-Lindau germline mutation V84L manifests as early-onset bilateral pheochromocytoma. Am J Med Genet A. 2006;140:685-690.

18. Grubb RL III, Choyke PL, Linehan WM, Walther MM. Management of von Hippel-Lindau-associated kidney cancer. Nat Clin Pract Urol. 2005;2:248-255.

19. Kovacs G: Molecular cytogenetics of renal cell tumour. Adv Cancer Res. 1993;62:89-124 
20. Birt AR, Hogg GR, Dube WJ. Hereditary multiple fibrofolliculomas with trichodiscomas and acrochordons. Arch Dermatol. 1997; 113:1674-1677.

21. Birt-Hogg-Dube gene (FLCN) causes dominantly inherited spontaneous pneumothorax. Am J Hum Genet. 2005;76:522527.

22. American Society of Clinical Oncology. ASCO policy statement update: genetic testing for cancer susceptibility. J Clin Oncol. 2003;21:2397-2406.

23. Linehan WM, Zbar B, Walther MM. The genetic basis of cancer of kidney cancer: implications for gene-specific clinical management. BJU Int 95 Suppl. 2005;2:2-7.

24. Da Silva NF, Gentle D, Maher ER. Analysis of the Birt-HoggDube (BHD) tumour suppressor gene in sporadic renal cell carcinoma and colorectal cancer. J Med Genet. 2003;40:820-4

25. Toro JR, Glenn G, Duray P, Linehan M, Turner ML. Birt-HoggDube syndrome: a novel marker of kidney neoplasia. Arch Dermatol. 1999; 135:1195-1202.

26. Nickerson ML, Toro JR, Merino M, Linehan WM, Zbar B, Schmidt LS. Mutations in a novel gene lead to kidney tumors, lung wall defects, and benign tumors of the hair follicle in patients with the Birt-Hogg-Dube syndrome. Cancer Cell. 2002;2:157-164.

27. Vocke CD, Yang Y, Pavlovich CP, Schmidt LS, Nickerson ML, Torres CA, Merino MJ, Zbar B, Linehan WM. High frequency of somatic frameshift BHD gene mutations in Birt-Hogg-Dubeassociated renal tumors. J Natl Cancer Inst. 2005; 97:931-939.

28. Schmidt LS, Toro JR, Merino MJ, Turner ML, Zbar B, Linehan WM. Germline BHD-mutation spectrum and phenotype analysis of a large cohort of families with Birt-Hogg-Dube syndrome. Am J Hum Genet. 2005;76:1023-1033.

29. Pavlovich CP, Grubb RL III, Linehan WM. Evaluation and management of renal tumors in the Birt-Hogg-Dube syndrome. J Urol. 2005; 173:1482-1486.

30. Launonen V, Vierimaa O, Kiuru M, Isola J, Roth S, Pukkala E, Sistonen P, Herva R, Aaltonen LA. Inherited susceptibility to uterine leiomyomas and renal cell cancer. Proc Natl Acad Sci U S A. 2001; 98:3387-3392

31. Alam NA, Tomlinson IP. Clinical features of multiple cutaneous and uterine leiomyomatosis: an underdiagnosed tumor syndrome. Arch Dermatol. 2005; 141: 199-206.

32. Alam NA, Rowan AJ, Wortham NC, Pollard PJ, Tomlinson IP. Genetic and functional analyses of $\mathrm{FH}$ mutations in multiple cutaneous and uterine leiomyomatosis, hereditary leiomyomatosis and renal cancer, and fumarate hydratase deficiency. Hum Mol Genet. 2003; 12: 1241-1252.

33. Kiuru M, Lehtonen R, Arola J, Salovaara R, Jarvinen H, Aittomaki K, Sjoberg J, Visakorpi T, Knuutila S, Aaltonen LA. Few FH mutations in sporadic counterparts of tumor types observed in hereditary leiomyomatosis and renal cell cancer families. Cancer Res. 2002; 62: 4554-4557.
34. Toro JR, Nickerson ML, Linehan WM, Schmidt LS, Zbar B. Mutations in the fumarate hydratase gene cause hereditary leiomyomatosis and renal cell cancer in families in North America. Am J Hum Genet. 2003; 73: 95-106.

35. Grubb RL 3rd, Franks ME, Toro J; et al: Hereditary leiomyomatosis and renal cell cancer: a syndrome associated with an aggressive form of inherited renal cancer.2007 Jun;177(6): 2074-2079.

36. Osborne JP, Fryer A, Webb D. Epidemiology of tuberous sclerosis. Ann N Y Acad Sci. 1991;615:125-127.

37. Al-Saleem T, Wessner LL, Henske EP. Malignant tumors of the kidney, brain and soft tissues in children and young adults with the TSC. Cancer 1998; 83:2208-2216.

38. Shepherd CW, Gomez MR, Lie JT, Crowson CS. Causes of death in patients with tuberous sclerosis. Mayo Clin Proc. 1991;66: 792-796.

39. European Chromosome 16 Tuberous Sclerosis Consortium. Identification of the tuberous sclerosis gene on chromosome 16. Cell. 1993;75:1305-1315.

40. Roach ES, Sparagana SP. Diagnosis of tuberous sclerosis complex. J Child Neurol. 2004;19:643-649.

41. Au KS, Williams AT, Gambello MJ, Northrup H. Molecular genetic basis of tuberous sclerosis complex: from bench to bedside. J Child Neurol. 2004; 19: 699-709.

42. Oesterling JE, Fishman EK, Goldman SM, Marshall FF. The management of renal angiomyolipoma. J Urol. 1986; 135: 1121-1124.

43. Pea M, Bonetti F, Martignoni G, Apparent renal cell carcinomas in tuberous sclerosis are heterogeneous: the identification of malignant epithelioid angiomyolipoma. Am J Surg Pathol. 1998;22:180-187.

44. Jones AC, Shyamsundar MM, Cheadle JP. Comprehensive mutation analysis of TSC1 and TSC2-and phenotypic correlations in 150 families with tuberous sclerosis. Am J Hum Genet. 1999; 64:1305-1315.

45. Sancak O, Nellist M, Mutational analysis of the TSC1 and TSC2 genes in a diagnostic setting: genotype-phenotype correlations and comparison of diagnostic DNA techniques in Tuberous Sclerosis Complex. Eur J Hum Genet. 2005;13:731-741.

Correspondencia autor: Dr. Julián Sanz-Ortega

Servicio de Oncología Médica

Hospital Clínico San Carlos

Prof. Martín Lagos, s/n - 28040 Madrid

Tel.: 913303822

Email autor: jsanzo.hcsc@salud.madrid.org

Información artículo: Revisión - Cáncer renal

Trabajo recibido: octubre 2008

Trabajo aceptado: noviembre 2008 